\title{
Maximum Echo-State-Likelihood Networks for Emotion Recognition
}

\author{
Edmondo Trentin ${ }^{1}$, Stefan Scherer ${ }^{2}$, and Friedhelm Schwenker ${ }^{2}$ \\ 1 Dipartimento di Ingegneria dell'Informazione, Università degli studi di Siena, \\ Siena, Italy \\ trentin@dii.unisi.it \\ 2 Institute of Neural Information Processing, Ulm University, Ulm, Germany \\ \{stefan.scherer, friedhelm.schwenker\}@uni-ulm.de
}

\begin{abstract}
Emotion recognition is a relevant task in human-computer interaction. Several pattern recognition and machine learning techniques have been applied so far in order to assign input audio and/or video sequences to specific emotional classes. This paper introduces a novel approach to the problem, suitable also to more generic sequence recognition tasks. The approach relies on the combination of the recurrent reservoir of an echo state network with a connectionist density estimation module. The reservoir realizes an encoding of the input sequences into a fixed-dimensionality pattern of neuron activations. The density estimator, consisting of a constrained radial basis functions network, evaluates the likelihood of the echo state given the input. Unsupervised training is accomplished within a maximum-likelihood framework. The architecture can then be used for estimating class-conditional probabilities in order to carry out emotion classification within a Bayesian setup. Preliminary experiments in emotion recognition from speech signals from the WaSeP C dataset show that the proposed approach is effective, and it may outperform state-of-the-art classifiers.
\end{abstract}

Keywords: Emotion recognition, echo state network, radial basis functions, maximum likelihood, density estimation.

\section{Introduction}

In the last few years, human-machine interaction (HMI) has been taking a more and more important position in our everyday life. However, up to date expressive and emotional conversation is a matter of human-human communication (HHC) only. Current machines are not capable of neither understanding nor expressing subjective states or emotional expressions. Since this second, implicit channel of communication (containing information about the speaker himself, the situation, and the ongoing interaction) is so important for natural and efficient HHC, it is believed that the only way to render HMI more natural and efficient is to implement the capabilities to recognize, understand, and express these conversational elements in machines. In the present work a novel approach towards recognizing emotional expressions is introduced. The model relies on the combination of an

F. Schwenker and N. El Gayar (Eds.): ANNPR 2010, LNAI 5998, pp. $60-71,2010$.
(C) Springer-Verlag Berlin Heidelberg 2010 
echo state network (ESN) [8] and a constrained radial basis functions (RBF)-like network [1] suitable for density estimation. The basic idea is that the recurrent reservoir of the ESN realizes an encoding of an input sequence (e.g., an acoustic observation sequence obtained from the speech signal of the user whose emotional state has to be recognized) by means of the pattern of activation of its state neurons. The RBF is trained in order to estimate the probability density function (pdf) which characterizes the distribution of the reservoir activation patterns within the encoding space. The model is trained according to an algorithm aimed at the maximization of the likelihood of the encoding (i.e., of the echo-state) given the input sequence. For these reasons, we refer to the overall machine as the maximum echo-state-likelihood network (MESLiN). The training scheme is inherently unsupervised and non-discriminative, along the line of statistical parametric pdf estimation techniques relying on the maximum-likelihood (ML) criterion 2]. Nonetheless, it can be applied in emotion recognition tasks by using a separate MESLiN for estimating the class-conditional pdf [2] for each of the classes involved in the problem. In order to proof the concept of this approach, experiments based on a corpus containing pseudo words spoken in six different emotional prosodies, WaSeP(C) [17, have been conducted.

The remainder of the paper is organized as follows. In Section 2 the approach is presented, introducing the echo state encoding and the ML estimation algorithm. Section 3 introduces the utilized dataset, the feature extraction process, and reports the performance of humans in a large scale perception test. Sections 4 and 5 report on the achieved classification results for the experiments and conclude the paper, respectively.

\section{The Maximum Echo-State-Likelihood Network}

The model is introduced in the framework of emotion recognition from speech signals, although it can be applied to several sequence recognition tasks using different feature spaces. As we stated in the previous section, a separate, classspecific, and independent MESLiN is used for each emotion involved in the task. In so doing, in the following we will focus on a generic machine, trained over the corresponding, emotion-specific training sample, with the understanding that the algorithm has to be subsequently applied to as many MESLiNs as the number of classes at hand.

In this perspective, suppose that a sample $\mathcal{T}=\left\{\mathcal{Y}_{1}, \ldots, \mathcal{Y}_{n}\right\}$ of $n$ acoustic observation sequences has been observed. The pdf estimation problem faced in this paper can be stated as follows: assuming that all the sequences in $\mathcal{T}$ have been independently drawn from a certain pdf $p(\mathcal{Y})$, how can the dataset be used in order to estimate a "reasonable" model of $p(\mathcal{Y})$ ? We assume that $p(\mathcal{Y})$ is a function having fixed and known parametric form, being determined uniquely by the specific value of a set of parameters $\theta=\left(\theta_{1}, \ldots, \theta_{k}\right)$. To render this dependency on $\theta$ in a more explicit manner, we will modify our notation slightly by writing $p(\mathcal{Y})$ as $p(\mathcal{Y} \mid \theta)$. Given the assumption, the formulation of the question posed above can be restated as: how can we use the sample $\mathcal{T}$ in order 
to obtain estimates for $\theta$ that are meaningful according to a certain "optimality" criterion? A sound answer to the question may be found in the adoption of the ML criterion, along with a suitable method for maximizing the likelihood $p(\mathcal{T} \mid \theta)$ of the parameters given the sample. Since $\mathcal{Y}_{1}, \ldots, \mathcal{Y}_{n}$ are assumed to be i.i.d., the likelihood $p(\mathcal{T} \mid \theta)$ can be written as $p(\mathcal{T} \mid \theta)=\prod_{i=1}^{n} p\left(\mathcal{Y}_{i} \mid \theta\right)$. Before attempting the maximization of the likelihood, it is necessary to specify a well-defined form for the pdf $p(\mathcal{Y} \mid \theta)$. Let us assume the existence of an integer $d$ and of two functions, $\phi:\{\mathcal{Y}\} \rightarrow \Re^{d}$ (where $\{\mathcal{Y}\}$ is the universe of all possible observation sequences) and $\hat{p}: \Re^{d} \rightarrow \Re$, s.t. $p(\mathcal{Y} \mid \theta)$ can be decomposed as:

$$
p(\mathcal{Y} \mid \theta)=\hat{p}(\phi(\mathcal{Y})) .
$$

It is seen that there exist (infinite) choices for $\phi($.$) and \hat{p}($.$) that satisfy Eq. (11),$ the most trivial being $\phi(\mathcal{Y})=p(\mathcal{Y} \mid \theta), \hat{p}(x)=x$. We call $\phi($.$) the encoding, while$ $\hat{p}($.$) is simply referred to as the "likelihood". Again, we assume parametric forms$ $\phi\left(\mathcal{Y} \mid \theta_{\phi}\right)$ and $\hat{p}\left(\mathbf{x} \mid \theta_{\hat{\mathbf{p}}}\right)$ for the encoding and for the likelihood, respectively, and we set $\theta=\left(\theta_{\phi}, \theta_{\hat{\mathbf{p}}}\right)$. For notational convenience, we will sometimes write $p(\mathcal{Y})$ as a shortcut for $p(\mathcal{Y} \mid \theta)$.

We propose a two-block connectionist/statistical model for $p(\mathcal{Y} \mid \theta)$ as follows. The function $\phi\left(\mathcal{Y} \mid \theta_{\phi}\right)$ is realized via an echo state network, suitable to map sequences $\mathcal{Y}$ into real vectors $\mathrm{x}$. The weights of the ESN become the parameters $\theta_{\phi}$. A radial basis functions (RBF)-like neural net is then used to model the likelihood function $\hat{p}\left(\mathbf{x} \mid \theta_{\hat{\mathbf{p}}}\right)$, where $\theta_{\hat{\mathbf{p}}}$ are the parameters of the RBF. In order to ensure that a pdf is obtained, constraints have to be placed on the hiddento-output connection weights of the RBF (assuming that normalized Gaussian kernels are used).

First, let us focus on the ESN-based model for $\phi\left(\mathcal{Y} \mid \theta_{\phi}\right)$. An ESN [8] is a particular, recent type of recurrent neural network (RNN). Among the advantages of an ESN over common RNNs are the stability towards noisy inputs [14] and the efficient method to adapt the weights of the network 7]. ESNs are applicable in many different tasks such as classification, pattern generation, or controlling tasks [1415/87]. However, in this application the ESN is used to encode the input data sequence within its current state, i.e. the pattern of activation of the neurons in its reservoir. A schematic representation of an ESN is shown in Figure 1. The most important part of the network is the so called reservoir. It is a collection of neurons (typically, from around ten to a few thousand in number), that are loosely connected to each other. Typically, the probability of a connection between neuron $a_{i}$ and neuron $a_{j}$ (to be set in the connection matrix $W$ ) is around $2 \%-10 \%$ and usually decreases with a rising number of neurons within the reservoir, whereas the connections between the input and output layer with the reservoir are all set. This loose connectivity leads, in turn, to several small cliques of neurons that are recursively connected to each other, sensitive to a certain dynamic within the data received through the input and from other connected neurons. If observed as separated from the rest of the network, an individual clique may appear to follow a seemingly random pattern. However, if observed along with all the competing and supporting cliques within the large 


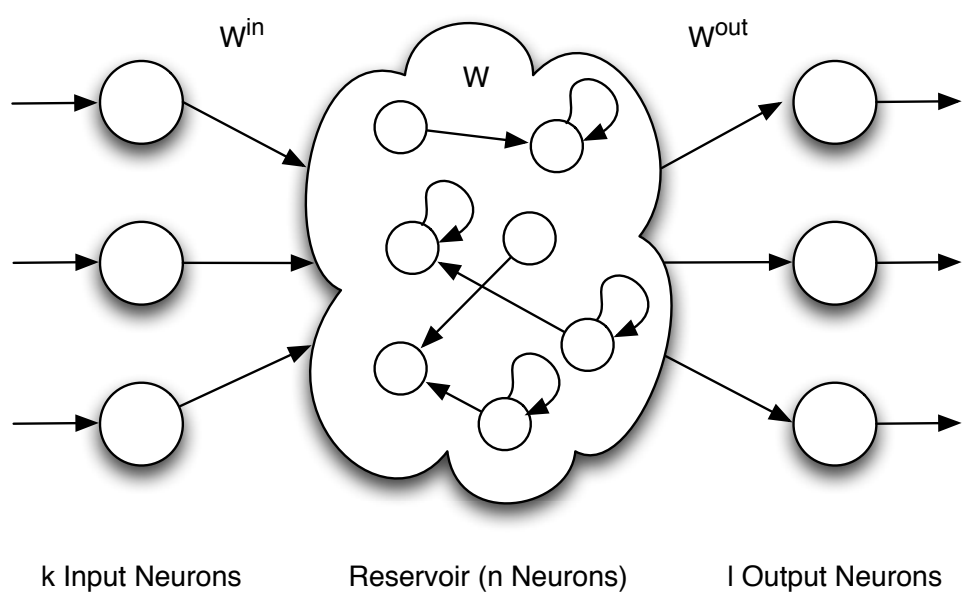

Fig. 1. Schematics of an echo state network with $k$ input neurons, $l$ output neurons, and $n$ neurons in the reservoir. The input is fully connected to the reservoir via $W^{i n}$ as well as the reservoir with the output via $W^{\text {out }}$. The topology of the connections within the reservoir, as well as their weights $W$, are set randomly.

reservoir, the reactions of the clique to the inputs are anything but random. Since there are feedback and recursive connections within the reservoir, not only the input is taken into account for the output but also the current state of each of the neurons, and the history of all previous inputs. Therefore, ESNs are an ideal candidate for encoding dynamic processes, such as emotional expressions or non-verbal utterances 1415 .

In contrast to standard feedforward neural networks, such as multi-layer perceptrons, the ESN incorporates previous features and states into its current state, rendering it an ideal approach for encoding tasks, possibly including the modeling of typical dynamics found in the speech signals (e.g., prosody of emotional expressions). The encoding of an acoustic observation sequence $\mathcal{Y}=\mathbf{y}_{1}, \ldots, \mathbf{y}_{T}$ (where $T$ is not fixed, but sequence-specific) for a generic ESN in the system is accomplished as follows:

1. Initialize the states of the ESN randomly.

2. Feed the ESN with the first $L$ acoustic feature vectors $\mathcal{Y}_{1}, \ldots, \mathcal{Y}_{L}$ (to minimize the influence of the random initialization) 1 .

3. Save the current state $\hat{\mathbf{x}}$ of the ESN as the starting state.

4. Set the ESN in $\hat{\mathbf{x}}$, and sequentially feed the ESN with inputs $\mathcal{Y}$.

5. Let $\mathbf{x}$ denote the encoding of $\mathcal{Y}$, i.e. the state of the reservoir at the end of sequence $\mathcal{Y}$.

6. Feed the RBF with $\mathbf{x}$.

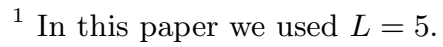


Given the fact that the weights $\theta_{\phi}$ of the ESN are not optimized, the ML estimation of the RBF parameters $\theta_{\hat{\mathbf{p}}}$ given $\mathcal{T}$ requires now to find parameters that maximize the quantity

$$
p\left(\mathcal{T} \mid \theta_{\hat{\mathbf{p}}}\right)=\prod_{i=1}^{n} \hat{p}\left(\phi\left(\mathcal{Y}_{i} \mid \theta_{\phi}\right) \mid \theta_{\hat{\mathbf{p}}}\right) .
$$

A hill-climbing algorithm to carry out ML estimation of the parameters $\theta_{\hat{\mathbf{p}}}$ can be obtained as an instance of the gradient-ascent method over $p\left(\mathcal{T} \mid \theta_{\hat{\mathbf{p}}}\right)$ in two steps: (i) initialization, i.e., start with some initial, e.g. "random", assignment of values to the RBF parameters; (ii) gradient-ascent, i.e., repeatedly apply a learning rule in the form $\Delta \theta_{\hat{\mathbf{p}}}=\eta \nabla_{\theta_{\hat{\mathbf{p}}}}\left\{\prod_{i=1}^{n} \hat{p}\left(\phi\left(\mathcal{Y}_{i} \mid \theta_{\phi}\right) \mid \theta_{\hat{\mathbf{p}}}\right)\right\}$ with $\eta \in \Re^{+}$. This is a batch learning setup. In practice, neural network learning may be simplified, yet even improved, with the adoption of an on-line training scheme that prescribes $\Delta \theta_{\hat{\mathbf{p}}}=$ $\eta \nabla_{\theta_{\hat{\mathbf{p}}}}\left\{\hat{p}\left(\phi\left(\mathcal{Y} \mid \theta_{\phi}\right) \mid \theta_{\hat{\mathbf{p}}}\right)\right\}$ upon presentation of each individual training sequence $\mathcal{Y}$. Two distinct families of adaptive parameters $\theta$ have to be considered:

(1) Mixing parameters $c_{1}, \ldots, c_{n}$, i.e. the hidden-to-output weights of the RBF network. Constraints have to be placed on these parameters during the ML estimation process, in order to ensure that they are in $[0,1]$ and that they sum to one. A simple way to satisfy the requirements is to introduce $n$ hidden parameters $\gamma_{1}, \ldots, \gamma_{n}$, which are unconstrained, and to set

$$
c_{i}=\frac{\varsigma\left(\gamma_{i}\right)}{\sum_{j=1}^{n} \varsigma\left(\gamma_{j}\right)}, i=1, \ldots, n
$$

where $\varsigma(x)=1 /\left(1+e^{-x}\right)$. Each $\gamma_{i}$ is then treated as an unknown parameter $\theta$ to be estimated via ML.

(2) $d$-dimensional mean vector $\mu_{i}$ and $d \times d$ covariance matrix $\Sigma_{i}$ for each of the Gaussian kernels $K_{i}(\mathbf{x})=N\left(\mathbf{x} ; \mu_{i}, \Sigma_{i}\right), i=1, \ldots, n$ of the RBF, where $N\left(\mathbf{x} ; \mu_{i}, \Sigma_{i}\right)$ denotes a multivariate Normal pdf having mean vector $\mu_{i}$, covariance matrix $\Sigma_{i}$, and evaluated over the random vector $\mathbf{x}$. A common (yet effective) simplification is to consider diagonal covariance matrices, i.e. independence among the components of the input vector $\mathbf{x}$. This assumption leads to the following three major consequences: (i) modeling properties are not affected significantly, according to [10; (ii) generalization capabilities of the overall model may turn out to be improved, since the number of free parameters is reduced; (iii) $i$-th multivariate kernel $K_{i}$ may be expressed in the form of a product of $d$ univariate Normal densities as:

$$
K_{i}(\mathbf{x})=\prod_{j=1}^{d} \frac{1}{\sqrt{2 \pi} \sigma_{i j}} \exp \left\{-\frac{1}{2}\left(\frac{x_{j}-\mu_{i j}}{\sigma_{i j}}\right)^{2}\right\}
$$

i.e., the free parameters to be estimated are the means $\mu_{i j}$ and the standard deviations $\sigma_{i j}$, for each kernel $i=1, \ldots, n$ and for each component $j=1, \ldots, d$ of the input space.

In the following, we will derive explicit formulations for $\frac{\partial \hat{p}\left(\phi\left(\mathcal{Y} \mid \theta_{\phi}\right) \mid \theta_{\hat{\mathbf{p}}}\right)}{\partial \theta}$ for the two families of free parameters $\theta$ within the proposed model. As regards a generic 
mixing parameter $c_{i}, i=1, \ldots, n$, from Eq. (3), and since $p(\mathcal{Y})=\sum_{k=1}^{n} c_{k} K_{k}(\mathbf{x})$, we have

$$
\begin{aligned}
\frac{\partial \hat{p}\left(\phi\left(\mathcal{Y} \mid \theta_{\phi}\right) \mid \theta_{\hat{\mathbf{p}}}\right)}{\partial \gamma_{i}} & =\sum_{j=1}^{n} \frac{\partial p(\mathcal{Y})}{\partial c_{j}} \frac{\partial c_{j}}{\partial \gamma_{i}} \\
& =\sum_{j=1}^{n} K_{j}(\mathbf{x}) \frac{\partial}{\partial \gamma_{i}}\left(\frac{\varsigma\left(\gamma_{j}\right)}{\sum_{k=1}^{n} \varsigma\left(\gamma_{k}\right)}\right) \\
& =K_{i}(\mathbf{x})\left\{\frac{\varsigma^{\prime}\left(\gamma_{i}\right) \sum_{k} \varsigma\left(\gamma_{k}\right)-\varsigma\left(\gamma_{i}\right) \varsigma^{\prime}\left(\gamma_{i}\right)}{\left[\sum_{k} \varsigma\left(\gamma_{k}\right)\right]^{2}}\right\}+\sum_{j \neq i} K_{j}(\mathbf{x})\left\{\frac{-\varsigma\left(\gamma_{j}\right) \varsigma^{\prime}\left(\gamma_{i}\right)}{\left[\sum_{k} \varsigma\left(\gamma_{k}\right)\right]^{2}}\right\} \\
& =K_{i}(\mathbf{x}) \frac{\varsigma^{\prime}\left(\gamma_{i}\right)}{\sum_{k} \varsigma\left(\gamma_{k}\right)}-\sum_{j} K_{j}(\mathbf{x}) \frac{\varsigma\left(\gamma_{j}\right) \varsigma^{\prime}\left(\gamma_{i}\right)}{\left[\sum_{k} \varsigma\left(\gamma_{k}\right)\right]^{2}} \\
& =K_{i}(\mathbf{x}) \frac{\varsigma^{\prime}\left(\gamma_{i}\right)}{\sum_{k} \varsigma\left(\gamma_{k}\right)}-\left\{\sum_{j} c_{j} K_{j}(\mathbf{x})\right\} \frac{\varsigma^{\prime}\left(\gamma_{i}\right)}{\sum_{k} \varsigma\left(\gamma_{k}\right)} \\
& =\frac{\varsigma^{\prime}\left(\gamma_{i}\right)}{\sum_{k} \varsigma\left(\gamma_{k}\right)}\left\{K_{i}(\mathbf{x})-p(\mathcal{Y})\right\} .
\end{aligned}
$$

For the means $\mu_{i j}$ and the standard deviations $\sigma_{i j}$ we proceed as follows. Let $\theta_{i j}$ denote the free parameter, i.e. $\mu_{i j}$ or $\sigma_{i j}$, to be estimated. It is seen that:

$$
\frac{\partial \hat{p}\left(\phi\left(\mathcal{Y} \mid \theta_{\phi}\right) \mid \theta_{\hat{\mathbf{p}}}\right)}{\partial \theta_{i j}}=c_{i} \frac{\partial K_{i}(\mathbf{x})}{\partial \theta_{i j}}
$$

where the calculation of $\frac{\partial K_{i}(\mathbf{x})}{\partial \theta_{i j}}$ can be accomplished as follows. First of all, let us observe that for any real-valued, differentiable function $f($.$) this property holds$ true: $\frac{\partial f(.)}{\partial x}=f(.) \frac{\partial \log [f(.)]}{\partial x}$. As a consequence, from Eq. (4) we can write

$$
\begin{aligned}
\frac{\partial K_{i}(\mathbf{x})}{\partial \theta_{i j}} & =K_{i}(\mathbf{x}) \frac{\partial \log K_{i}(\mathbf{x})}{\partial \theta_{i j}} \\
& =K_{i}(\mathbf{x}) \frac{\partial}{\partial \theta_{i j}} \sum_{k=1}^{d}\left\{-\frac{1}{2}\left[\log \left(2 \pi \sigma_{i k}^{2}\right)+\left(\frac{x_{k}-\mu_{i k}}{\sigma_{i k}}\right)^{2}\right]\right\} .
\end{aligned}
$$

For the means, i.e. $\theta_{i j}=\mu_{i j}$, Eq. (17) yields

$$
\frac{\partial K_{i}(\mathbf{x})}{\partial \mu_{i j}}=K_{i}(\mathbf{x}) \frac{x_{j}-\mu_{i j}}{\sigma_{i j}^{2}} .
$$

For the covariances, i.e. $\theta_{i j}=\sigma_{i j}$, Eq. (17) takes the form:

$$
\begin{aligned}
\frac{\partial K_{i}(\mathbf{x})}{\partial \sigma_{i j}} & =K_{i}(\mathbf{x}) \frac{\partial}{\partial \sigma_{i j}}\left\{-\frac{1}{2} \log \left(2 \pi \sigma_{i j}^{2}\right)-\frac{1}{2}\left(\frac{x_{j}-\mu_{i j}}{\sigma_{i j}}\right)^{2}\right\} \\
& =\frac{K_{i}(\mathbf{x})}{\sigma_{i j}}\left\{\left(\frac{x_{j}-\mu_{i j}}{\sigma_{i j}}\right)^{2}-1\right\} .
\end{aligned}
$$




\section{Dataset Description and Feature Extraction}

The experiments in this work are based on the "Corpus of spoken words for studies of auditory speech and emotional prosody processing" (WaSePC) [17], which consists of two main parts: a collection of German nouns and a collection of phonetically balanced pseudo words, which correspond to the phonetical rules of German language. For this study the pseudo words have been chosen as the basis. This pseudo word set consists of 222 words, repeatedly uttered by a male and a female actor in six different emotional prosodies: neutral, joy, sadness, anger, fear, and disgust. Furthermore, a perception test has been conducted with 74 native German listeners, who were asked to rate and name the category or prosody that they were just listening to, resulting in an overall accuracy of $78.53 \%$. It was also observed that the most confused emotion is "disgust", which is conform with the assumptions of Scherer [13.

In the dataset, each of the pseudo words consists of a concatenation of two syllables, including for instance: "hebof", "kebil", or "sepau". The average duration of the speech signals depends on the specific emotion, ranging from 0.75 sec. in the case of the "neutral" prosody, to $1.70 \mathrm{sec}$. in the case of "disgust". Figure 2 shows a sample waveform (and, spectra) of a signal from the dataset, corresponding to the emotion "joy". The data was recorded using a Sony TCD-D7 DAT-recorder and the Sennheiser MD 425 microphone in an acoustic chamber with a $44.1 \mathrm{kHz}$ sample rate and later down-sampled to $16 \mathrm{kHz}$ with a 16 bit resolution. Relative Spectral Perceptual Linear Predictive Coding (RASTA-PLP) acoustic parameters were used as acoustic features. In [4] perceptual linear predictive speech analysis (PLP) was first introduced as a method to represent speech signals with respect to human perception and with as few parameters as possible. However, PLP is sensitive to steady-state spectral factors caused by transmission channels, e.g. different transducers [6 5]. Therefore, 6] proposes the relative spectral methodology (RASTA) for PLP, rendering it more robust without increasing the computational burden significantly.

The PLP analysis is based on two perceptually and biologically motivated concepts, namely the critical bands, and the equal loudness curves, shown in Figure 3. One line represents the sound pressure $(\mathrm{dB})$ that is required to perceive a sound of any frequency as loud as a reference sound of $1 \mathrm{kHz}$. The critical band filtering is analogous to the popular Mel frequency scaled cepstral coefficients (MFCC) triangular filtering, but the 21 filters are equally spaced along the Bark scale (instead of applying the Mel scale). The equal loudness curve is approximated by

$$
E(w)=1.151 \cdot \sqrt{\frac{\left(w^{2}+144 \cdot 10^{4}\right) \cdot w^{2}}{\left(w^{2}+16 \cdot 10^{4}\right) \cdot\left(w^{2}+961 \cdot 10^{4}\right)}},
$$

according to [12, and it is applied to the filtered signal. The next steps are specific to the RASTA processing, and they follow the implementation recommendations in 6]. After transforming the spectrum to the logarithmic domain and the application of RASTA filtering, the signal is transformed back using 

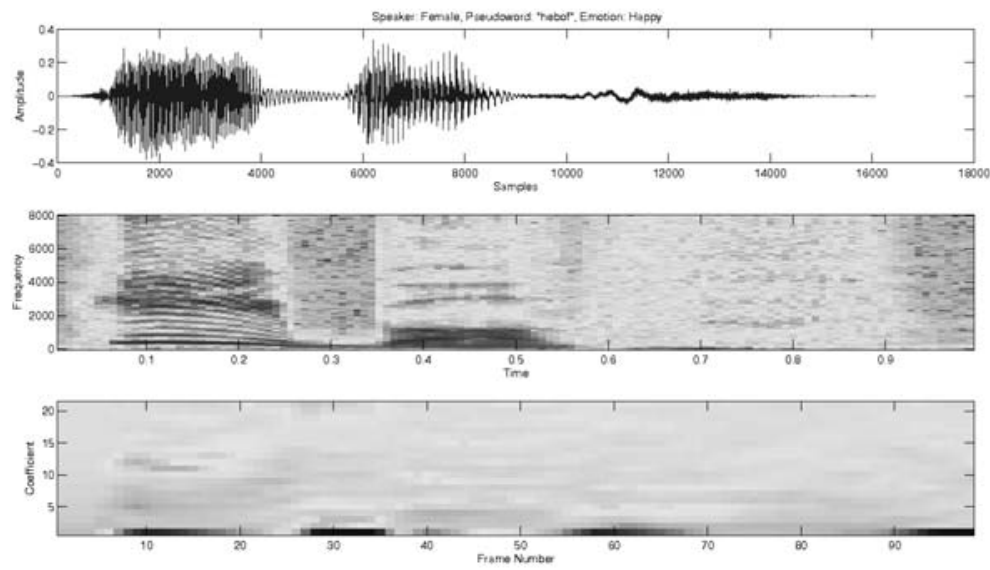

Fig. 2. Original waveform (top), spectrum after RASTA processing (middle), and RASTA-PLP cepstral coefficients (bottom)

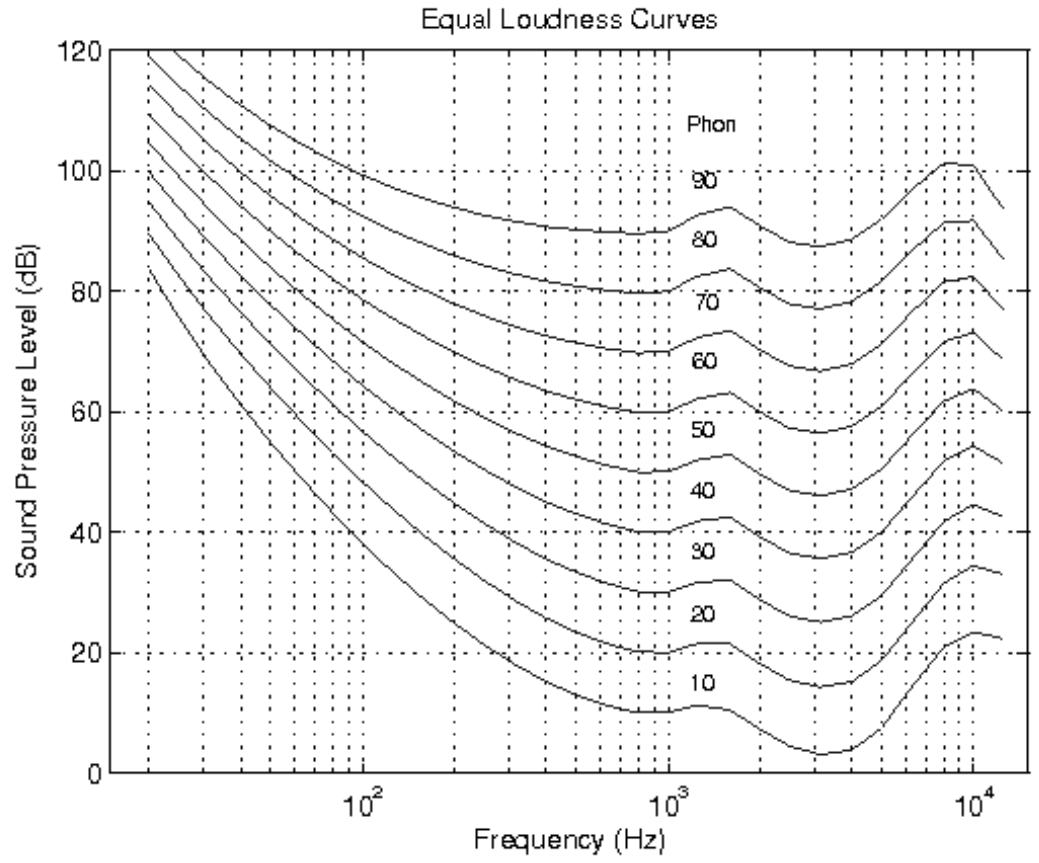

Fig. 3. Equal loudness curves

the exponential function. The last steps are comprised by an estimation of the linear predictive coding (LPC) coefficients as introduced by [11], and by the transformation of the LPC coefficients to cepstral values. In Figure 2 a comparison between the original waveform, the spectrum after RASTA processing and the RASTA-PLP cepstral coefficients are shown. 


\section{Experiments}

Preliminary experiments were carried out using female speech signals from the WaSeP(C) dataset, for a total of 1386 variable-length acoustic observation sequences (231 sequences per each class of emotion). A 10-fold crossvalidation procedure has been adopted for evaluating the classification performance. Each fold was defined by splitting the overall dataset, at random, into a training sample (1254 sequences) and a test sample (132 sequences). The folds were created such that (i) the 10 test sets did not overlap with each other (i.e., no sequences could appear more than once in a test set), and (ii) a uniform prior distribution of individual classes was granted (namely, 22 sequences per class in each test set). Unfortunately, no direct competition from related work is available for the dataset used in this experiment. A qualitative baseline is the result from the human perception test mentioned in Section 3 (average accuracy of $78 \%$ ). Furthermore, similar results (around 70\%) are available in the literature. For example, $70 \%$ accuracy was achieved in a seven category experiment using three feature sets in a multi classifier system [16. In the earlier work by Lee et al., similar frame-based features, as in this approach, were used as input to hidden Markov models (HMM). MFCC-based features were extracted and the classification task was to identify four different emotions. The HMMs reached an overall accuracy of around $76 \%[9]$.

Table 1 reports the recognition accuracies (averaged over the 10 folds) obtained with MESLiN, and other traditional classifiers, in the present setup. All the traditional classifiers were trained at the acoustic frame level. Classification on test set was accomplished by averaging over the class-specific scores yielded by the classifier along the whole observation sequence. The nearest neighbor classifier 2] was used first, in order to fix a baseline. Then, Multi-layer Perceptron (MLP) networks were trained, having 2-layer topology featuring a hidden layer with 14 units, sigmoid activation functions in both hidden and output layers. Training was accomplished by applying 500 training epochs with a learning rate of 0.3 , and a momentum rate equal to 0.2 (these parameters were selected via preliminary cross-validation). The same MLP architecture, and training parameters, were also applied within a 10-iterations AdaBoost strategy 3], Support vector machine (SVM) with RBF kernels were used, having $\gamma=0.1, \epsilon=0.001$ (tolerance of the termination criterion.), and $\nu=0.5$.

Table 1. Average recognition accuracy of sequences from the WaSeP(C dataset

\begin{tabular}{ll}
\hline \hline Method & Average accuracy (\%) \\
\hline Nearest Neighbor & 33.90 \\
MLP & 39.32 \\
AdaBoost & 45.87 \\
SVM & 48.01 \\
MESLiN & 86.39 \\
\hline
\end{tabular}


Finally, MESLiN was evaluated. Due to the intrinsically non-discriminative, ML training setup used in this paper, separate class-specific MESLiNs were trained independently over the training sequences belonging to the corresponding class. Classification was accomplished according to the usual maximum-aposteriori (MAP) framework, via Bayes decision rule [2]. Since the class prior probabilities are identical in the present setup, the MAP criterion reduces to a direct maximum class-conditional pdf decision rule, i.e. assign a sequence to the emotion whose MESLiN model yields the highest likelihood. The MESLiNs are initialized as follows. Since the output of the feature extraction process described in Section 3 is 21-dimensional, $k=21$ input neurons are used for the reservoir. Furthermore, they are connected to all the $n=100$ state neurons (with tanh as transfer function) in the reservoir, featuring randomly initialized topology and values of the connection weights. An overall $10 \%$ of the possible unit-to-unit connections in the reservoir are taken, and the weight matrix $W$ is normalized with a spectral width of $\alpha=1$ [7]. The RBF-like network features $n=3$ kernels, having mean, covariances, and mixing parameters initialized as follows. The components of the mean vectors were initialized at random (uniformly) over the range $\mathcal{I}=(-0.5,0.5)$; the components of the diagonal covariance matrices were initialized to a fixed value, namely $\sqrt{|\mathcal{I}| / n}$; the mixing parameters were initialized at random over the interval $(0.0,1.0)$ such that they sum to 1 . Training of the MESLiN was accomplished for 20 epochs, using different, quantity-specific learning rates for the mixing parameters $\left(\eta_{\gamma}=1.0 e-06\right)$, the means of the Gaussians $\left(\eta_{\mu}=1.0 e-10\right)$, and the corresponding variances $\left(\eta_{\sigma}=1.0 e-11\right)$. All the parameters were set during a preliminary cross-validation stage. Results confirm the approach is effective. A concise discussion is given in the next section. Table 2 shows the confusion matrix yielded by MESLiN. It is seen that "disgust" is the most confusable class. This is in line with the same phenomenon reported above for the human listeners. In this case, it is confused with "neutral" most of the times.

Table 2. Confusion matrix (MESLiN)

\begin{tabular}{|c|c|c|c|c|c|c|}
\hline & neutral & joy & $\operatorname{sadn}$ & orer & fear & liso \\
\hline neutral & 21.90 & 0.10 & 0.00 & 0.00 & 0.00 & 0.00 \\
\hline joy & 0.50 & 18.70 & 0.20 & 0.00 & 2.50 & 0.10 \\
\hline sadness & 0.50 & 0.00 & 21.40 & 0.10 & 0.00 & 0.00 \\
\hline anger & 0.00 & 1.10 & 0.30 & 18.90 & 0.30 & 1.40 \\
\hline fear & 0.00 & 0.60 & 1.60 & 0.20 & 19.60 & 0.00 \\
\hline disgust & 14.80 & 0.90 & 0.60 & 0.10 & 2.00 & 3.60 \\
\hline
\end{tabular}

\section{Conclusions}

The paper introduced a novel connectionist approach to the task of sequence modeling and classification. The model combines the reservoir of an ESN with a constrained RBF-like architecture. The former realizes a recurrent encoding 
of the observation sequence into a pattern of activation of the neurons (i.e., the state of the ESN), whilst the RBF estimates the pdf of such encodings. A MLbased training scheme was developed, which ensures satisfaction of probabilistic axioms (i.e., the estimated model turns out to be a proper pdf). Training is inherently unsupervised (the pdf of the overall training sample is estimated). Nonetheless, the machine can be successfully applied to supervised classification tasks over sequences, by training separate class-conditional models for each one of the classes involved in the problem. In so doing, each class features its own recurrent encoding ESN, and its own pdf model. The algorithm was applied to emotion recognition in speech signals from the WaSePC dataset. Preliminary results confirm the approach is effective, yielding the highest recognition accuracies over a number of state-of-the-art classifiers. Surprisingly enough, the performance of the proposed classifier is even higher than the average accuracy yielded by humans on a similar task. We argue this is due to the following reasons: (i) the system was trained on data having the same nature as the data used for test, whilst humans do not undergo any data-specific training, but they assign test utterances to an emotional class according to generic, prior knowledge of their (subjective) concept of specific emotions; (ii) in particular, from the humans' point if view, no hard distinctions can be made between certain emotions; and (iii) the audio recordings in the dataset are not real-world utterances, since they were performed by actors. It is likely that this introduces a significant bias, such that humans cannot easily recognize the emotion from the acted expression, while the machine learns from the training sample how actors tend to give a certain interpretation of the same emotion (e.g., affecting specific features) that, later, can be easily recognized in the test data. In the light of these considerations, future work is focusing on a fully unsupervised application of the proposed model: sequences belonging to all classes are merged in a single training set, and the ML training is applied in order to let the machine discover its own "clusters" of emotions (i.e., how the emotions are distributed and concentrated in the encoding space yielded by the ESN).

\section{Acknowledgment}

The collaboration of this work would not have been possible without the generous funding of the Vigoni program, supported by DAAD, MIUR and by Ateneo Italo-Tedesco (AIT). Furthermore, the presented work was developed within the Transregional Collaborative Research Centre SFB/TRR 62 "CompanionTechnology for Cognitive Technical Systems" funded by the German Research Foundation (DFG).

\section{References}

1. Bishop, C.M.: Neural Networks for Pattern Recognition. Oxford University Press, Oxford (1995)

2. Duda, R.O., Hart, P.E.: Pattern Classification and Scene Analysis. Wiley, New York (1973) 
3. Freund, Y., Schapire, R.E.: Experiments with a new boosting algorithm. In: Proceedings of the Thirteenth International Conference on Machine Learning, San Francisco, pp. 148-156 (1996)

4. Hermansky, H., Hanson, B., Wakita, H.: Perceptually based linear predictive analysis of speech. In: IEEE International Conference on Acoustics, Speech, and Signal Processing, ICASSP 1985, April 1985, vol. 10, pp. 509-512 (1985)

5. Hermansky, H., Morgan, N., Bayya, A., Kohn, P.: Rasta-plp speech analysis. Technical report, ICSI Technical Report TR-91-069 (1991)

6. Hermansky, H., Morgan, N., Bayya, A., Kohn, P.: Rasta-plp speech analysis technique. In: IEEE International Conference on Acoustics, Speech, and Signal Processing, ICASSP 1992, vol. 1, pp. 121-124 (1992)

7. Jaeger, H.: Tutorial on training recurrent neural networks, covering bppt, rtrl, ekf and the echo state network approach. Technical Report 159, FraunhoferGesellschaft, St. Augustin Germany (2002)

8. Jaeger, H., Haas, H.: Harnessing nonlinearity: Predicting chaotic systems and saving energy in wireless communication. Science 304, 78-80 (2004)

9. Lee, C.M., Yildirim, S., Bulut, M., Kazemzadeh, A., Busso, C., Deng, Z., Lee, S., Narayanan, S.S.: Emotion recognition based on phoneme classes. In: Proceedings of ICSLP 2004 (2004)

10. McLachlan, G.J., Basford, K.E. (eds.): Mixture Models: Inference and Applications to Clustering. Marcel Dekker, New York (1988)

11. Rabiner, L.R.: Fundamentals of Speech Recognition. Prentice-Hall, Englewood Cliffs (1993)

12. Robinson, D.W., Dadson, R.S.: A re-determination of the equal-loudness relations for pure tones. British Journal of Applied Physics 7(5), 166-181 (1956)

13. Scherer, K.R., Johnstone, T., Klasmeyer, G.: Vocal expression of emotion. In: Davidson, R.J., Scherer, K.R., Goldsmith, H.H. (eds.) Handbook of Affective Sciences, Affective Science, pp. 433-456. Oxford University Press, Oxford (2003)

14. Scherer, S., Oubbati, M., Schwenker, F., Palm, G.: Real-time emotion recognition from speech using echo state networks. In: Prevost, L., Marinai, S., Schwenker, F. (eds.) ANNPR 2008. LNCS (LNAI), vol. 5064, pp. 205-216. Springer, Heidelberg (2008)

15. Scherer, S., Schwenker, F., Campbell, W.N., Palm, G.: Multimodal laughter detection in natural discourses. In: Proceedings of 3rd International Workshop on Human-Centered Robotic Systems, HCRS 2009 (2009)

16. Scherer, S., Schwenker, F., Palm, G.: Classifier fusion for emotion recognition from speech. In: 3rd IET International Conference on Intelligent Environments 2007 (IE 2007), pp. 152-155. IEEE, Los Alamitos (2007)

17. Wendt, B., Scheich, H.: The magdeburger prosodie korpus - a spoken language corpus for fmri-studies. In: Speech Prosody 2002, SProSIG (2002) 